\title{
Variación estructural de la comunidad perifítica colonizadora de sustratos artificiales en la zona de ritral del río Medellín, Colombia
}

\author{
Yimmy Montoya M. ${ }^{1}$ \& John J. Ramírez R. ${ }^{1,2}$ \\ 1 Departamento de Biología, Universidad de Antioquia. Apartado 1226, Medellín, Cra 31 No 28-11, Colombia. Fax: \\ 57-4-2330120, yimmymontoya3@hotmail.com \\ 2 jjram@matematicas.udea.edu.co
}

Recibido 08-VII-2005. Corregido 18-X-2006. Aceptado 30-I-2007.

\begin{abstract}
Variation in the structure of the periphytic community that colonizes artificial substrata in the Medellín river (Colombia). Weekly samples were taken from microscope glass slides attached to acrylic substrata were placed at subsuperficial level in two sampling stations in the higher section of the Medellín river, Colombia, from January through March 1998. Diversity and evenness varied with time (Friedman $\mathrm{p}<0.05$ ). There were spatial differences in biomass, productivity, diversity and evenness (Friedman $\mathrm{p}<0.05$ ). Total biomass and productivity increased with the time of colonization at station 1 . Station 2, with four times more water flow, had lower diversity and mean evenness values. During the colonization process the were 76 species (22 families). The Chrysophyta, mainly diatoms, were the most abundant (56\% of the total), followed by Clorophyta (29\%), Cyanophyta (11\%) and Cryptophyta (4 \%). Rev. Biol. Trop. 55 (2): 585-593. Epub 2007 June, 29.
\end{abstract}

Key words: Periphyton, stream, biofilms, colonization, tropical limnology.

El perifiton se define como una comunidad compleja de microbiota (algas, bacterias, hongos, animales, detritos orgánicos e inorgánicos) adherida a un sustrato, que puede ser orgánico o inorgánico, vivo o muerto (Wetzel 1983). Según Marshall (1984), se designa como biofilm o biopelícula al agregado de microorganismos y productos extracelulares asociados a un sustrato. Debido a que el muestreo cuantitativo en sustratos naturales es difícil, los sustratos artificiales son bastante utilizados, sobre todo en experimentos de colonización.

La importancia que en general se atribuye al perifiton en los ecosistemas acuáticos fue resumida por Moreira (1988) quien destacó su producción de metabolitos orgánicos que alimentan diversos organismos; su contribución con más del $70 \%$ de la materia orgánica a la productividad total; sus altas tasas de reciclaje; su posibilidad de proporcionar abrigo y alimento a varios tipos de organismos, principalmente peces; su alta productividad primaria y su papel como mejor indicador biológico que el fitoplancton.

En Colombia, los pocos estudios realizados sobre colonización han sido llevados a cabo principalmente en ecosistemas lénticos (Moreno 1989, Donato et al. 1996, Sierra y Ramírez 2000). Investigaciones sobre la comunidad perifítica presente en los ríos son abordados principalmente para estudios de calidad de aguas e impacto ambiental, basados en raspados sobre sustratos naturales en distintas estaciones de muestreo en donde la zona alta de los ríos es usada como una estación patrón o control. El propósito de esta investigación es mostrar si la comunidad perifítica colonizadora de sustratos artificiales varía en su composición, distribución, diversidad de especies, productividad y biomasa debido la influencia de las variaciones de la descarga, ligadas a su vez a las mudanzas en el régimen hidrológico 
en la zona de muestreo, en un intervalo de ocho semanas en dos estaciones de investigación en el tramo alto del río Medellín.

\section{MATERIALES Y MÉTODOS}

La cuenca del río Medellín se encuentra ubicada dentro de la reserva ecológica del alto de San Miguel $\left(6^{\circ} 03^{\prime} \mathrm{N}\right.$ y $\left.75^{\circ} 37^{\prime} \mathrm{W}\right)$ entre los 1800 y $2800 \mathrm{msnm}$ sobre la Cordillera Central, al sur del municipio de Caldas en el Departamento de Antioquia, Colombia. El clima de la zona es de régimen tropical de montaña, con temperatura promedio anual variando de 12 a $20{ }^{\circ} \mathrm{C}$ y precipitación anual oscilando entre 2 000-3 500 mm (Anónimo 1982). El área de estudio corresponde según Espinal (1964), a la zona de vida bosque muy húmedo Montano Bajo (bmh-MB). Esta zona se encuentra situada en el ecosistema de alta montaña denominado "bosque de niebla".

Se ubicaron dos estaciones separadas por una distancia aproximada de $50 \mathrm{~m}$ y la primera estación presentó un caudal cuatro veces mayor que la segunda. Los caudales se evaluaron a partir de la evaluación batimétrica del cauce y la evaluación de la velocidad por transectos de $20 \mathrm{~cm}$ usando un medidor de flujo global FP-101. En cada estación se colocaron dos muestreadores de acrílico con 25 portaobjetos, a una profundidad entre 5 y $10 \mathrm{~cm}$ debajo de la superficie del río en el centro de su cauce. Su ubicación en el lecho del río fue vertical en relación con la corriente, según lo recomendado por Sladeckova (1962) y Wetzel (1983). El tiempo de exposición fue de ocho semanas, en concordancia con lo establecido por Lobo y Buselato (1985) y con exposiciones preliminares en el sitio de muestreo. La toma de muestras se inició el 30 de enero/98 y se realizaron recoleccións semanales hasta completar ocho semanas de muestreo (27 de marzo/98).

Se estimaron in situ diferentes variables hidroclimáticas, físicas y químicas tales como radiación solar, temperatura del aire, humedad relativa, precipitación, caudal, conductividad eléctrica, sólidos totales, turbidez, oxígeno disuelto, $\mathrm{pH}$, alcalinidad, dureza, fósforo soluble reactivo, nitritos, nitratos, nitrógeno amoniacal y carbono inorgánico disuelto (Montoya y Ramírez, en prep.). Para la recolección del perifiton de cada estación de muestreo se retiraron tres placas al azar; una para el análisis de clorofila $a$ siendo colocada directamente en acetona al $95 \%$ basificada a $\mathrm{pH} 8$ según las recomendaciones de Wetzel y Likens (1991); otra placa se empleó para determinar la productividad de la comunidad, la cual se estimó mediante el método de acumulación de biomasa propuesto por APHA (1995). La placa restante se empleó para el análisis cualitativo y cuantitativo de los taxónes ficoperifíticos presentes, por lo que fue fijada con lugol $\left(\mathrm{KI}-\mathrm{I}_{2}\right)$ en el campo. En el laboratorio las placas fueron raspadas con una cuchilla fina y se procedió a identificar los organismos hasta el máximo nivel taxonómico posible, empleando para ello material bibliográfico específico (Krammer y Lange-Bertalot 1986, 1988, 1991a, b, Patrick 1966, 1975). El conteo se realizó en la placa de colonización, en un aumento de 40X. Se tomaron tres transectos a lo largo de la placa, los cuales fueron elegidos aleatoriamente. Los datos de densidad son presentados como individuos por metro cuadrado utilizando una variación de la fórmula citada en Ross (1979) para conteos con base en franjas.

Para el análisis de la comunidad de diatomeas, se procedió a lavar la muestra con HCL al $10 \%$ durante $5 \mathrm{~min}$. Después se lavó con agua destilada tres veces y se centrifugó a $1500 \mathrm{rpm}$ por $3 \mathrm{~min}$. Posteriormente, se oxidó la materia orgánica con peróxido de hidrógeno a $50{ }^{\circ} \mathrm{C}$ por $24 \mathrm{~h}$. Finalmente, se montaron las placas permanentes en NAPHRAX (Moro 1998), las cuales fueron depositadas en la colección de referencia del laboratorio de limnología de la Universidad de Antioquia.

Los datos obtenidos fueron sometidos a análisis exploratorio mediante el uso de estadísticos de tendencia central (media aritmética, MA) y de dispersión (desviación estándar y coeficiente de variación relativa de Pearson, $\mathrm{CV})$. Para establecer la significancia de las diferencias entre estaciones de muestreo se 
utilizó la prueba no paramétrica de Friedman (Díaz 1999).

Para explicar el comportamiento de las variables estudiadas a nivel temporal y espacial, se empleó el coeficiente de correlación de Spearman, $r_{s}$. Se evaluó la estructura de la comunidad mediante curvas de importancia y usando los índices de dominancia (Simpson 1949), diversidad (Shannon y Weaver 1949) y equidad (Pielou 1969). Adicionalmente, se realizó una prueba de correlación múltiple entre la estructura de la comunidad y las variables fisicoquímicas empleándose el programa Statgraphics versión 5.0.

\section{RESULTADOS}

Durante el proceso de colonización se encontraron 76 especies pertenecientes a 22 familias (Cuadro 1), siendo la división crisofita, principalmente las diatomeas las más abundantes con un $56 \%$ del total, seguida por las clorofíceas con el $29 \%$, luego por las cianofíceas con el $11 \%$ y finalmente por las criptofíceas con el $4 \%$, las cuales sólo se informaron en la estación 1 .

Entre las variables fisicoquímicas estudiadas a nivel temporal únicamente la pluviosidad presentó diferencias significativas entre los periodos de muestreo (Friedman $\mathrm{p}<0.05$ ) y a nivel espacial se encontró que el caudal, el oxígeno disuelto, el FSR y el N-NH${ }_{4}$ exhibieron diferencias significativas (Friedman $\mathrm{p}<0.05$ ). Las demás variables estudiadas presentaron rangos de variación bajos en todas las dimensiones, lo cual es reflejo de la uniformidad química del terreno (Cuadro 2).

Entre los índices comunitarios estudiados, en la dimensión temporal se encontraron diferencias significativas en la diversidad y en la equidad (Friedman $\mathrm{p}<0.05$ ); a nivel espacial se encontraron diferencias en la biomasa, la productividad, la diversidad y la equidad (Friedman $\mathrm{p}<0.05$ ).

La figura 1A y B presenta la estructura de la comunidad perifítica colonizadora de sustratos artificiales durante el tiempo de investigación, observándose un proceso rápido de colonización (figura 2) que muestra la variación de los valores de la diversidad, equidad, riqueza numérica de especies y el caudal en las dos estaciones de muestreo. La comunidad perifítica de la estación 1 presentaron la menor concentración de clorofila a activa, de biomasa y productividad $\left(0.54 \mathrm{mg} \mathrm{C} \mathrm{m}^{-2}, 5.54 \mathrm{mg} \mathrm{m}^{-2}\right.$ y $\left.0.16 \mathrm{mg} \mathrm{C} \mathrm{m}^{2} \mathrm{dí}^{-1}\right)$, respecto a la comunidad colonizadora en la estación $2\left(3.04 \mathrm{mg} \mathrm{C} \mathrm{m}^{-2}\right.$, $14.29 \mathrm{mg} \mathrm{m}^{-2}$ y $0.55 \mathrm{mg} \mathrm{C} \mathrm{m}^{2}$ día $\left.^{-1}\right)$, la cual presentó valores entre tres y seis veces mayores.

\section{DISCUSIÓN}

Las dos estaciones presentaron caudales muy diferentes (Fig. 1C), lo que implica un arrastre diferencial de sedimentos, variación en la capacidad de dilución y en la dinámica del río. Experimentos en el laboratorio han demostrado que existen efectos fisiológicos de la corriente sobre el perifiton, ya que Stigeoclonium y Oedogonium dominaron la comunidad perifítica cuando el flujo se mantuvo en $9 \mathrm{~cm} / \mathrm{s}$, mientras que una masa densa de diatomeas domina la comunidad cuando la corriente muestra una velocidad de $38 \mathrm{~cm} / \mathrm{s}$ (McIntire 1973). La estación 1 presentó un caudal cuatro veces mayor que el de la estación 2, lo cual implica que los organismos de la comunidad ficoperifítica están sometidos a condiciones de flujo diferenciales, lo que finalmente repercutió sobre $\mathrm{su}$ estructura. Gomphonema subclavatum var mexicanun presentó una abundancia del 65.7 $\%$ en la estación 1 y en la estación 2 presentó un valor muy inferior con $6.84 \%$, lo que podría implicar que esta diatomea se desarrolla mejor en condiciones de mayor caudal.

El proceso de colonización, como lo muestra la figura 3, presentó un patrón consistente en una etapa inicial, en la que ocurre una fase de rápida colonización de especies, alcanzándose la máxima diversidad entre la segunda y tercera semana de colonización y la máxima riqueza en la cuarta semana del proceso de colonización. A partir de esta semana, los procesos de interacción entre las especies se hacen más fuertes 
CUADRO 1

Lista del perifiton encontrado en el Río Medellín

TABLE 1

Checklist of periphyton foun in the Medellin river

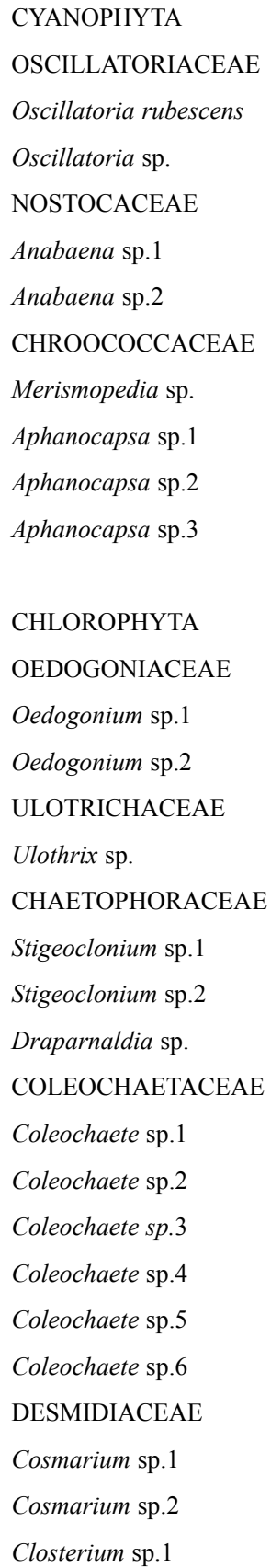

Closterium sp.2

Closterium sp. 3

Closterium sp.4

Pleurotaenium cf trabecula

OOCYSTACEAE

Selenastrum sp.1

Selenastrum $\mathrm{sp} .2$

CLADOPHORACEAE

Dermatophyton sp.

CRYPTOPHYTA

CRIPTOMONADACEAE

Cryptomona sp.1

Cryptomona sp.2

CHRYSOPHYTA

HETEROCLONIACEAE

Heterococcus sp.1

CRIPTOMONADACEAE

Cryptomona sp.1

Cryptomona sp.2

Fragilaria capucina var capucina

Fragilaria capucina var vaucheria

Fragilaria capucina var rumpens

Tabellaria floculosa

Tabellaria fenistrata

EUNOTIACEAE

Eunotia bilunaris

Eunotia denticulata

Eunotia hemyciclus

Eunotia minor

Eunotia monodom

Eunotia paludosa

Eunotia soleroi

ACHNANTHACEAE

Achnanthes inflata
Achnanthes lanceolata

Achnanthes minutisima var inconspicua

Achnanthes minutisima var gracilima

Achnanthes minutisima var minutisima

Cocconeis placentula

GOMPHONEMACEAE

Gomphonema acuminatum

Gomphonema subclavatum var mexicanum

Gomphonema clavatum

Gomphonema parvulum

NAVICULACEAE

Diploneis eliptica

Frustulia romboides

Frustulia vulgaris

Navicula criptocephala

Navicula cuspidata

Navicula mutica

Navicula subrhynchocephala

Pinnularia maior

Pleurosigma angulatum

Stauroneis phoenicenterum

CYMBELLACEAE

Cymbella mesiana

Cymbella minuta

EPHITEMIACEAE

Rhopalodia gibberula

NITZSCHIACEAE

Nitzschia palea

COSCINODISCACEAE

Melosira varians

Melosira granulata

THALASSIOSIRACEAE

Cyclotella $\mathrm{sp} .1$

Cyclotella sp.2 
CUADRO 2

Análisis estadísticos de las variables estudiadas

TABLE 2

Statistical analysis of the studied variables

\begin{tabular}{|c|c|c|}
\hline VARIABLE & F - RADIO & $\begin{array}{l}\text { NIVEL DE } \\
\text { SIGNIFICA }\end{array}$ \\
\hline \multicolumn{3}{|l|}{ Diversidad } \\
\hline Estaciones & 8.198 & $0.0063 *$ \\
\hline Tiempo & 8.274 & $0.0238 *$ \\
\hline \multicolumn{3}{|l|}{ Equidad } \\
\hline Estaciones & 8.578 & $0.0055 *$ \\
\hline Tiempo & 8.592 & $0.0220 *$ \\
\hline \multicolumn{3}{|l|}{ Riqueza } \\
\hline Estaciones & 2.161 & $0.1654 \mathrm{~ns}$ \\
\hline Tiempo & 0.234 & $0.6451 \mathrm{~ns}$ \\
\hline \multicolumn{3}{|l|}{ Biomasa } \\
\hline Estaciones & 3.301 & 0.0689 n.s \\
\hline Tiempo & 6.603 & $0.0370 *$ \\
\hline \multicolumn{3}{|l|}{ Clorofila a } \\
\hline Estaciones & 1.233 & $0.3947 \mathrm{~ns}$ \\
\hline Tiempo & 4.814 & $0.0643 \mathrm{~ns}$ \\
\hline \multicolumn{3}{|c|}{ Productividad } \\
\hline Estaciones & 2.504 & $0.1245 \mathrm{~ns}$ \\
\hline Tiempo & 6.202 & $0.0416 *$ \\
\hline
\end{tabular}

aumentando la competencia, ya que el espacio comienza a ser un factor limitante, sumado a otras variaciones, como el aumento en las precipitaciones y las variaciones del caudal. Hacia el final del periodo de colonización, aumentaron la biomasa total y la productividad, relacionados con los altos valores medios de nitrógeno $(7.98 \mathrm{mg} / \mathrm{l})$ y fósforo $(0.08 \mathrm{mg} / \mathrm{l})$ que no se pueden considerar como limitantes de la producción.

La correlación de Spearman entre la diversidad y la equidad, presentaron correlación en ambas estaciones (Spearman $\mathrm{p}<0.05$ ); esto implica que en las comunidades ficoperifíticas estudiadas, la diversidad depende principalmente de la equidad, ya que las especies dominantes se presentan en menor proporción con respecto a las especies raras, las cuales presentan una gran cola en la distribución de taxónes encontrados durante el proceso de colonización (Fig. 2). En la misma figura se observa que la estructura de la comunidad entre las estaciones de muestreo muestra diferencias, ya que la estación presentó cuatro especies que alcanzan el $83 \%$ de la abundancia total: G. sub. var mexicanum $65.74 \%$, Achanthes lanceolata $8.37 \%$, Navicula cuspidata 5.37 \% y Melosira varians $3.44 \%$. Estas especies se encontraron durante todo el proceso de colonización, y presentan secreciones mucilaginosas, tamaños pequeños y rápida multiplicación, por lo que se consideran estrategas $\mathrm{R}$.

La estructura de la comunidad de la estación 2 , presentó un valor medio de dominancia menor que el de la estación 1 , con un valor de 0.16 y 0.27 respectivamente, ya que se presentan seis especies con una abundancia relativa que alcanza el $83 \%$ de la abundancia total, entre las 66 especies encontradas a lo largo de la colonización, siendo las dominantes: Achanthes minutisima var minutisima con $26.98 \%$, N. cuspidata con $26.08 \%$, Fragilaria capucina var capucina con $8.9 \%$, Gomphonema parvulum con $6.97 \%$, G. sub. var mexicanum con $6.84 \%$ y finalmente, $A$. lanceolata con $6.45 \%$.

La variación de la relación productividad primaria/biomasa total en la comunidad de la estación 1 presentó oscilaciones bruscas y mayores que en la estación 2 , con un valor medio de 0.24 y 0.27 respectivamente, lo que implicaría que por cada g.C. $\mathrm{m}^{-2}$ obtenido a través de la fotosíntesis, estuvo involucrada una biomasa mayor para producirlo en la estación 1. Al principio los organismos colonizadores presentan una eficiencia fotosintética y un biovolumen alto (M. varians, Stigeoglonium sp., F. cap. var capucina, G. sub. var mexicanum y Pleurotaenium cf trabecula), pero con el paso del tiempo de colonización disminuyó la eficiencia de la comunidad en general.

La razón D430 / D665 presentó un valor medio de 2.25 para la estación 1 y de 2.22 para 

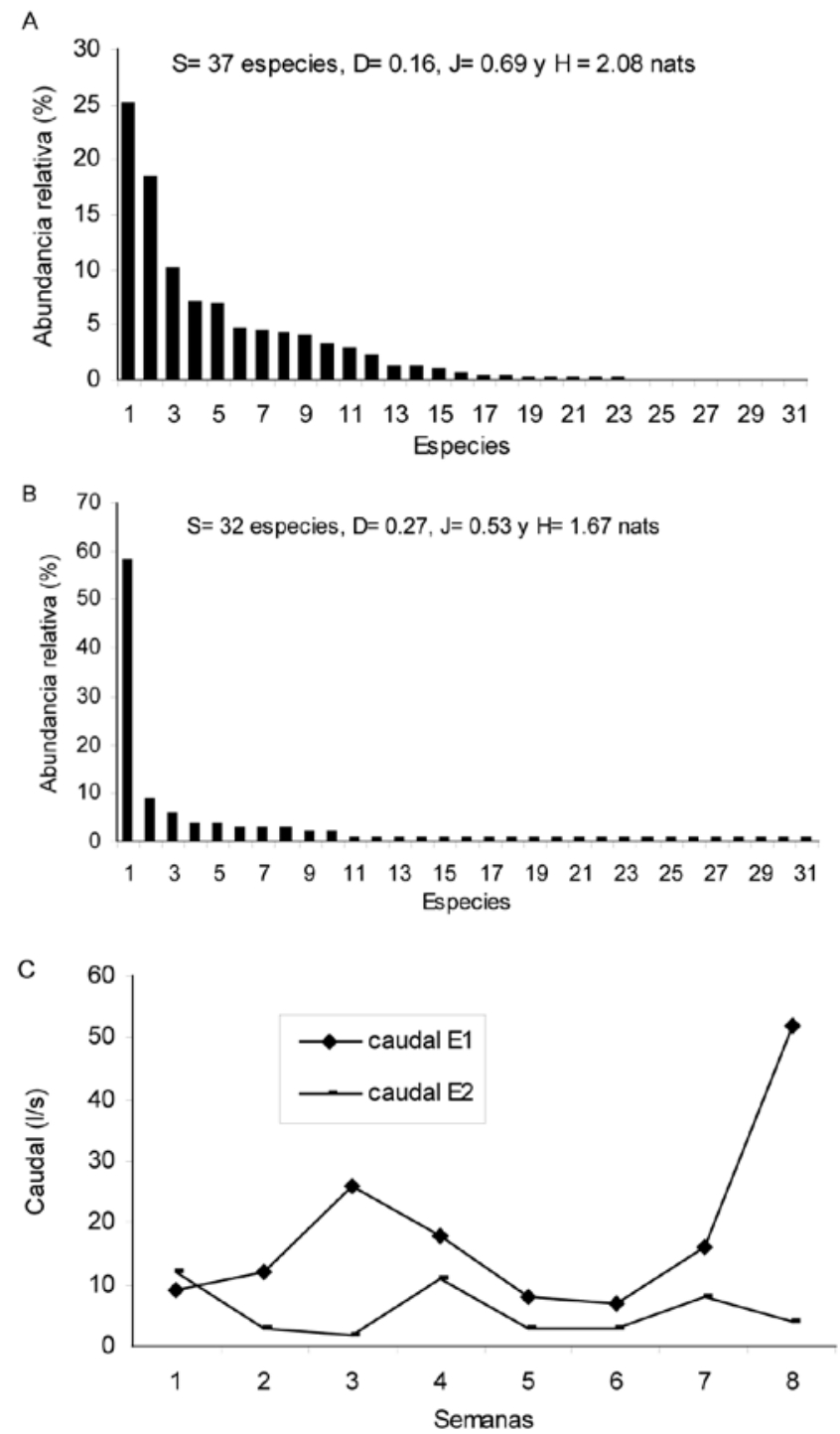

Fig. 1. Estructura de la comunidad ficoperifítica colonizadora de sustratos artificiales en la zona de ritral del río Medellín (S $=\mathrm{N}^{\mathrm{o}}$ de taxónes, $\mathrm{D}=$ Dominancia, $\mathrm{J}=$ Equidad y $\mathrm{H}=$ Diversidad) A. Estación 1. B. Estación 2. C. Comparación del caudal de las estaciones 1 y 2 .

Fig. 1. Structure of the phycoperiphytic community colonized artificial substrata in the ritral zone of the Medellín river (S $=\mathrm{N}^{\mathrm{o}}$ de taxa, $\mathrm{D}=$ Dominancy, $\mathrm{J}=$ evenness and $\mathrm{H}=$ Diversity). A. Station 1. B. Station 2. C. Stream velocity comparation between stations 1 and 2 . 

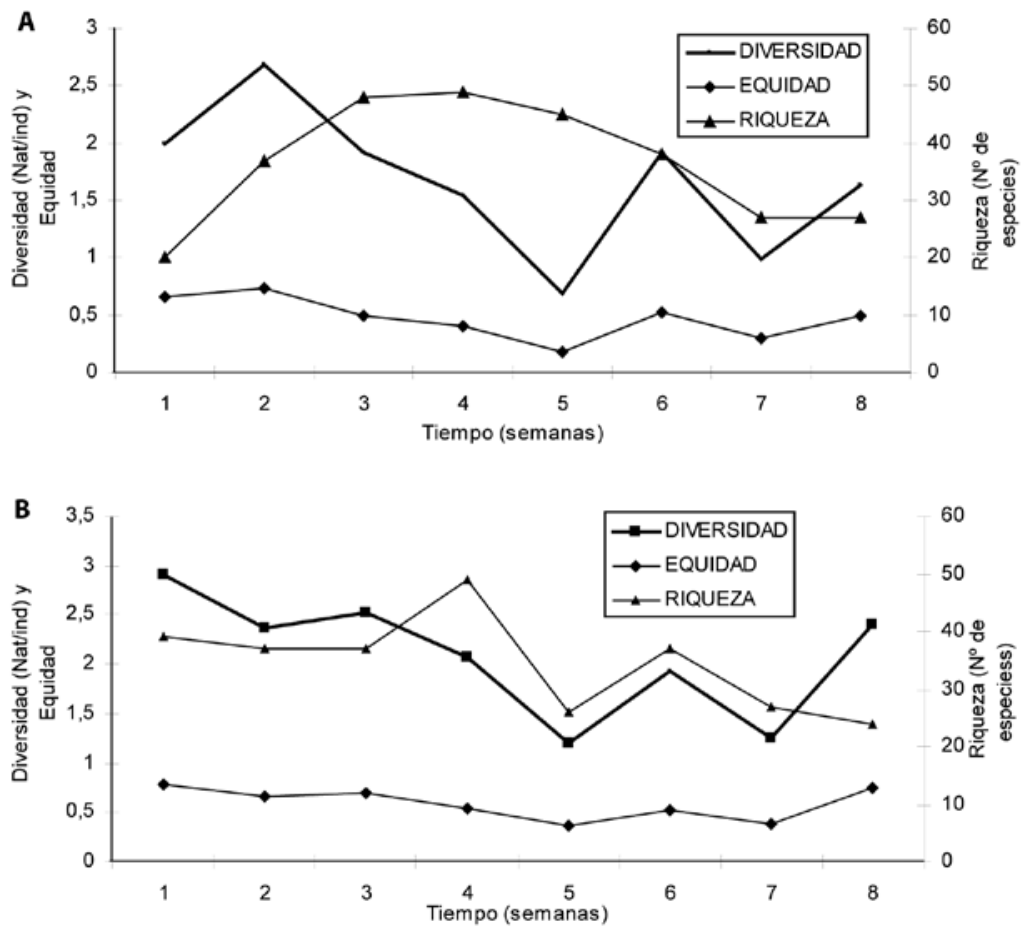

Fig. 2. Variación de la diversidad, la equidad, la riqueza numérica de especies y el caudal durante el proceso de colonización del ficoperifiton en la zona de ritral del río Medellín. A. Estación 1. B. Estación 2.

Fig. 2. Variation of the diversity, the evenness, the numeric wealth of species and the flow during the process of colonization of the ficoperifiton in the ritral zone of the Medellín river. A. Station 1. B. Station 2.

la estación 2, lo que implicaría que los carotenoides presentaron en general, dos veces la concentración de clorofila $a$ activa. Estos valores se presentan dentro del ámbito de 2.5 a 3.3, el cual es característico de sistemas bajo estrés (Margalef 1983). Este índice presentó el menor valor $(0.3$ y 0.32$)$ en la estación 1 y 2 respectivamente, en la última semana de muestreo, lo cual implica una tasa de renovación alta, lo cual pudo haber tenido relación con el aumento en las precipitaciones y caudales.

Las especies que fueron seleccionadas como descriptores ecológicos según las recomendaciones de Moro (1998); (A. lanceolata, A. min. var minutisima, N. cuspidata, F. cap. var capucina, $G$. sub. var mexicanum, $G$. parvulum y $M$. varians), han sido informadas ampliamente como componentes del perifiton. De las especies anteriores, sólo $M$. varians pertenece al orden centrales, siendo propiamente bentónica (Margalef 1983).

Las variables ambientales, fisicoquímicas y biológicas estudiadas en este trabajo, se presentaron bajo unas condiciones muy particulares de alta radiación solar, baja precipitación, amplitud en las fases de desecación; las cuales presentaron una comunidad ficoperifítica particular, la cual no necesariamente sería la típica de la zona, ya que esta se caracteriza por unas condiciones ambientales muy contrastantes con las que se presentaron en la realización de este experimento.

La comunidad ficoperifítica pese a presentar variaciones espacio-temporales, mostró un amplio predominio de las diatomeas, las cuales desde el proceso de colonización hasta el final del periodo de investigación, se caracterizaron por presentar el mayor número de especies y 

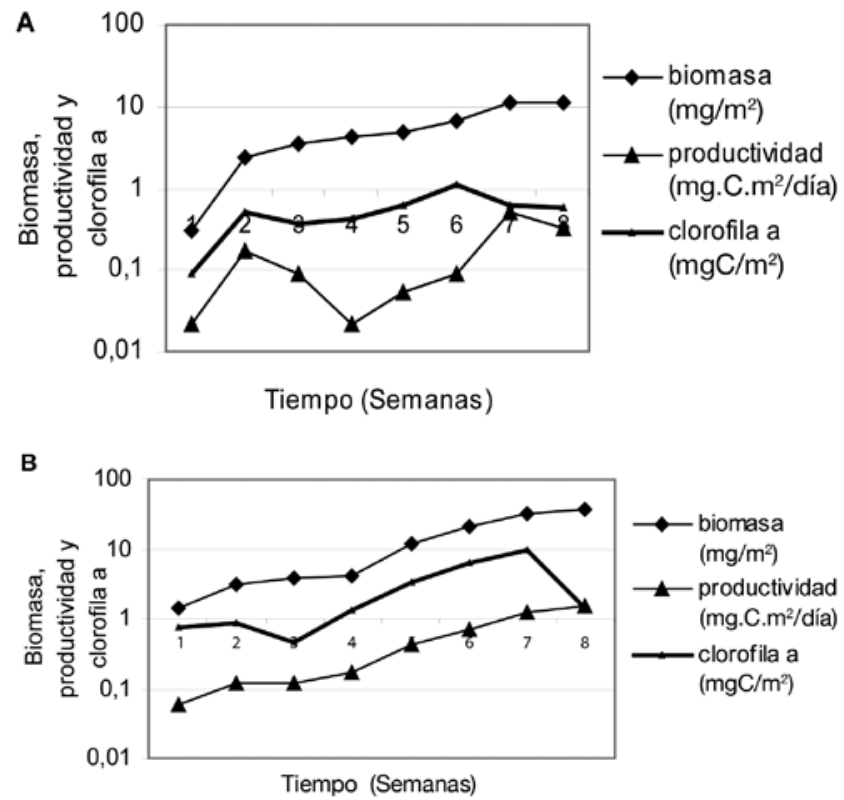

Fig. 3. Variación de la biomasa, la clorofila a activa y la productividad ficoperifítica durante ocho semanas de estudio en la zona de ritral del río Medellín. A. Estación 1. B. Estación 2.

Fig. 3. Variation of the biomass, the active chlorophyll and the phycoperiphytic productivity during eight weeks of study in the ritral zone of the Medellin river. A. Station 1. B. station 2.

por registrar variaciones respecto al caudal. Este comportamiento podría presentar cambios al realizar muestreos en otra época más característica de la zona lo cual se hace muy difícil debido a las continuas crecidas del río.

\section{AGRADECIMIENTOS}

El presente estudio está enmarcado dentro del proyecto "Caracterización preliminar del Alto de San Miguel" financiado por el Instituto Mi Río en convenio con la Universidad de Antioquia. Agradecemos la colaboración taxonómica de Rosemeri Segecin Moro la cual facilitó la ejecución de este trabajo.

\section{RESUMEN}

Se colocaron muestreadores de material acrílico con portaobjetos a nivel subsuperficial en dos estaciones de recolección en la parte alta del río Medellín. De enero a marzo de 1998 se tomaron muestras semanales y se encontraron diferencias temporales significativas en la diversidad $\mathrm{y}$ en la equidad (Friedman $\mathrm{p}<0.05$ ). A nivel espacial hubo diferencias en la biomasa, la productividad, la diversidad y la equidad (Friedman $\mathrm{p}<0.05)$ ). La biomasa total y la productividad aumentaron con el tiempo de colonización en la estación 1. La estación 2, que tiene un caudal cuatro veces mayor, tuvo menor diversidad y equidad media. Durante el proceso de colonización se encontraron 76 especies pertenecientes a 22 familias, siendo la división de las crisofitas, principalmente diatomeas, la más abundante $(56 \%$ del total), seguida por clorofíceas (29\%), cianofíceas (11\%) y criptofíceas (1 $4 \%$ ).

Palabras clave: perifiton, ríos, biopelícula, colonización, limnología tropical.

\section{REFERENCIAS}

Anónimo. 1982. Monografía del río Medellín. Falta la editorial o casa responsable, Medellín, Colombia. $44 \mathrm{p}$.

APHA, AWWA \& WEF. 1995. Standart Methods for the examination of water and wastewaters. United Book, Baltimore, Maryland, EEUU. 986p. 
Botero, G. 1963. Contribución al conocimiento de la zona central de Antioquia. An. Fac. Minas 57: 36-75.

Díaz, A. 1999. Diseño estadístico de experimentos. Universidad de Antioquia, Medellín, Colombia. $347 \mathrm{p}$.

Donato, R.J., G.E. Gonzáles \& M.L. Rodríguez. 1996. Ecología de dos sistemas acuáticos de páramo. Universidad Javeriana, Santafé de Bogotá, Colombia. $164 \mathrm{p}$.

Espinal, S. 1964. Formaciones vegetales de Antioquia. Rev. Fac. Nal. Agron. 24: 60-67.

Krammer, K. \& H. Lange-Bertalot. 1986, 1988, 1991a, 1991b. 4 v. Süsswasserflora von Mitteleuropa. Bacillariophyceae. Stuttgart, Alemania.

Lobo, E. \& C. Buselato. 1985. Tempo de exposicao de um substrato artificial para o establecimiento da comunidad do perifíton no curso inferior do rio Caí, rio grande do sul, Brasil. Rickia 12: 35-51.

Margalef, R. 1983. Limnología. Omega, Barcelona, España. 951 p

McIntire, C.D. 1973. Periphyton dynamics in laboratory streams: a simulation model and its implications. Ecol. Monogr. 43: 399-420.

Moreira, J.A. 1988. Productividad primaria do periphyton em viveiros destinados a piscicultura. Disertação do Mestrado, Univ. Federal de Bahia,Bahía, Brasil. 250 p.

Moreno, L.F. 1989. Colonización del perifiton en tres embalses del oriente Antioqueño. Tesis de pregrado, Universidad de Antioquia, Medellín, Colombia. $130 \mathrm{p}$.
Moro, S.R. 1998. Interpretacoes Peloecológicas Do Quaternário A través Da Análise De Diatomáceas (Bacillariophyta) Nos Sedimentos Da Lagoa Dourada, Ponta Grosa. Pr. Tese, Universidade Estadual Paulista, Sao Paulo, Brasil. 220p.

Patrick R. \& W.C. Reimer. 1966. The diatoms of United States. Acad. Nat. Sci. Philadelphia, vol 1, 688 p.

Patrick R. \& W.C. Reimer. 1975. The diatoms of United States. Acad. Nat. Sci. Philadelphia, vol 2, 213 p.

Pielou, E.C. 1969. Shannon's formula as a measure of specific diversity-its use and disue. Am. Nat. 100: 463-465.

Ross, J. 1979. Prácticas de ecología. Omega, Barcelona, España. 235 p.

Sierra, O. \& J. Ramírez. 2000. Variación espacio - temporal de biopelículas en la represa La Fe, el Retiro, Antioquia (Colombia). Actual Biol. 22: 153-168.

Sladeckova, A. 1962. Limnological investigation methods for the periphyton ("Aufwuchs") community. Bot. Rev. 28: 236-350.

Shannon, C.E. \& W. Weaver. 1949. The mathematical theory of communication. Illinois, Urbana, EEUU. $117 \mathrm{p}$.

Simpson, E.H. 1949. Measurement of diversity. Nature 163: 698 .

Wetzel, R.G. (ed.) 1983. Periphyton of aquatic ecosystem. B.V. Junk, The Hague, Holanda. 346 p.

Wetzel, R.G. \& E. Likens. 1991. Limnological Analyses. Springer-Verlag, Nueva York, EEUU. 391 p. 
